\title{
Recent Achievements of Plasma Studies within PF-1000U Facility
}

\author{
M.J. SAdowski ${ }^{a, b, *}$, M. PAdUCH ${ }^{b}$, P. KuBES ${ }^{c}$, \\ V.I. KraUZ ${ }^{d}$ AND V.A. GriBKOV ${ }^{e}$ \\ ${ }^{a}$ National Centre for Nuclear Research (NCBJ), 05-400 Otwock-Swierk, Poland \\ ${ }^{b}$ Institute of Plasma Physics and Laser Microfusion (IPPLM), 01-497 Warsaw, Poland \\ ${ }^{c}$ Czech Technical University (CVUT), 166-27 Prague, Czech Republic \\ ${ }^{d}$ National Research Centre, Kurchatov Institute, 123182 Moscow, Russia \\ ${ }^{e}$ Institute of Metallurgy and Materials Science, 119334 Moscow, Russia
}

Doi: $10.12693 /$ APhysPolA.138.613

*e-mail: marek.sadowski@ncbj.gov.pl

\begin{abstract}
This paper presents the most important results of recent plasma studies performed within the PF-1000U facility at the IPPLM in Warsaw, Poland. It was shown that a conical-tip of the anode facilitates the pinch formation and induces an increase in the total neutron yield, while the use of an anti-anode shortens the pinch column but does not improve the fusion-neutron emission. Experiments confirmed that the initial gas conditions have a strong influence on plasma parameters and, at some conditions, the local electron temperatures can reach high values ranging between 5 and $8 \mathrm{keV}$. It was shown that $\mathrm{PF}-1000 \mathrm{U}$ discharges with the $\mathrm{H}_{2}$-filling generate intense proton beams which can be used for research on $\left(\mathrm{p}^{-11} \mathrm{~B}\right)$ fusion reactions. It was also demonstrated that at the appropriate gas puffing the PF-1000U facility generates long plasma jets which can be used for simulations of astrophysical phenomena. Research on interactions of intense plasma streams with different solid targets showed that the facility can be useful for material engineering, e.g. for studies of plasma facing components of a future thermonuclear reactor DEMO. Measurements of fast deuteron beams provided information about their angular distribution and energy spectra. The evolution of a pinch column during the emission of charged particle beams was also observed and analyzed.
\end{abstract}

topics: plasma-focus, PF-1000U facility, anti-anode, pinch formation, total neutron yield, plasma jets

\section{Introduction}

The PF-1000U facility is one of the largest Mather-type plasma-focus machines in the world. In fact, this machine (initially called the PF-1000 facility) was put into full operation at a megajoule level in 2000 [1, 2]. From the very beginning, the machine has been equipped with a stainlesssteel vacuum chamber of $1.8 \mathrm{~m}$ in diameter and $3.8 \mathrm{~m}$ in length. At one end, it is closed by big current-collector plates connected through highvoltage coaxial cables with a large 1-MJ condenser bank which can be charged to $40 \mathrm{kV}$ but is usually operated up to $23 \mathrm{kV}, 330 \mathrm{~kJ}$ [3-5]. Inside the vacuum chamber, the collector plates are also connected with coaxial electrodes of $46 \mathrm{~cm}$ in length. These electrodes were changed during early experiments but in the present configuration, the outer grounded electrode (cathode of $40 \mathrm{~cm}$ in diameter) is composed of 12 stainless-steel tubes (each of $8 \mathrm{~cm}$ in diameter), distributed symmetrically around the periphery. The inner electrode (anode) is a thick-wall copper tube fixed inside a tubular ceramic insulator (of $15 \mathrm{~cm}$ in length).

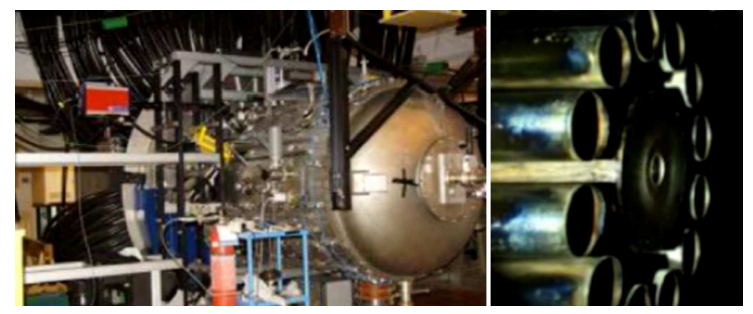

Fig. 1. General view of the PF-1000U chamber and outlets of typical electrodes (in a different scale). In the hole of the anode end-plate a nozzle of the pulsed gas valve can be seen.

The anode outlet is closed by a thick end-plate with a central hole which is used for fixing a nozzle of a pulsed gas-valve or special end-tips. Pictures of the PF-1000U facility and electrode outlets are shown in Fig. 1.

Plasma discharges in the PF-1000 (and recently in the PF-1000U), carried out in the energy range from about $200 \mathrm{~kJ}$ to about $1 \mathrm{MJ}$ and discharge currents reaching 1.4-1.8 MA, have been investigated for many years [3-11]. Various experiments 
have been performed under the auspices of the International Centre for Dense Magnetised Plasmas (ICDMP) and as part of national or international grants. The main aim of this paper is to summarize the most important achievements of several experimental campaigns performed in two recent years.

\section{Influence of the anode end and an anti-anode}

In order to investigate an influence of the anode ending on plasma dynamics and emission characteristics, a conical copper tip fixed at the centre of the anode end-plate was used. In 2016, different tips were applied and tested during an experimental campaign at the PF-1000U facility [12]. These tests showed that the radial collapse of the current sheath can be changed and that during a pinch formation a dense copper-plasma jet is formed along the $z$-axis, as shown in Fig. 2.

Changes in plasma dynamics and emission characteristics were confirmed not only by the recorded $\mathrm{X}$-ray frames but also by laser interferometric images and measurements with scintillation probes. Therefore, the experiments with the conical tips upon the PF-1000U anode end were continued in 2017 by means of different diagnostic techniques [13]. Some exemplary interferometric images of a pinch column are presented in Fig. 3 .

Here, it can be concluded that the conical tip eliminates the plasma penetration into the anodechannel, changes a distribution of the electric field and facilitates the pinch. It also decreases the dimensions of the pinch column by $\approx 30 \%$ and reveals a connection of the X-ray and neutron production with the second current dip. At appropriate experimental conditions, it is possible to obtain an increase in the fusion neutron yield to about $10^{11}$ neutrons from a $300 \mathrm{~kJ}$ shot.

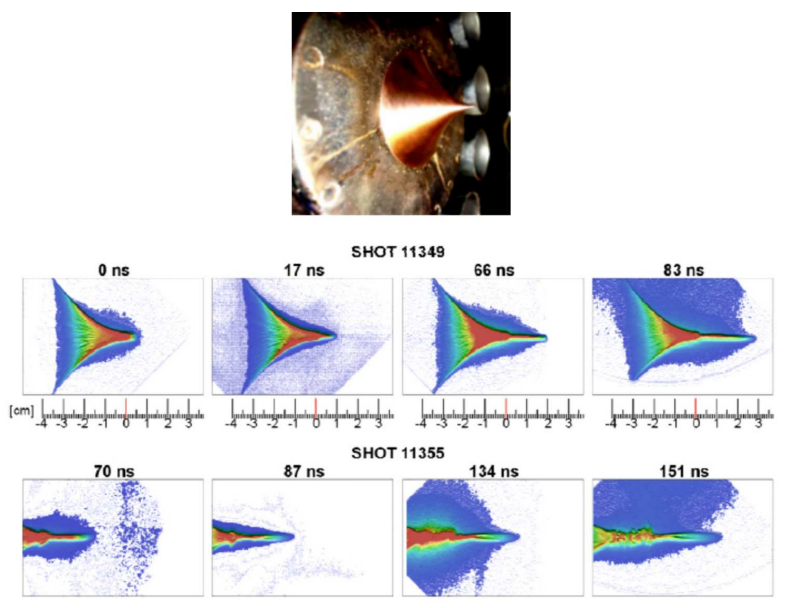

Fig. 2. Picture of the anode end with a conical tip and colour-enhanced X-ray images taken sideon at different instants of two shots which illustrate the formation and disintegration of plasma jets.

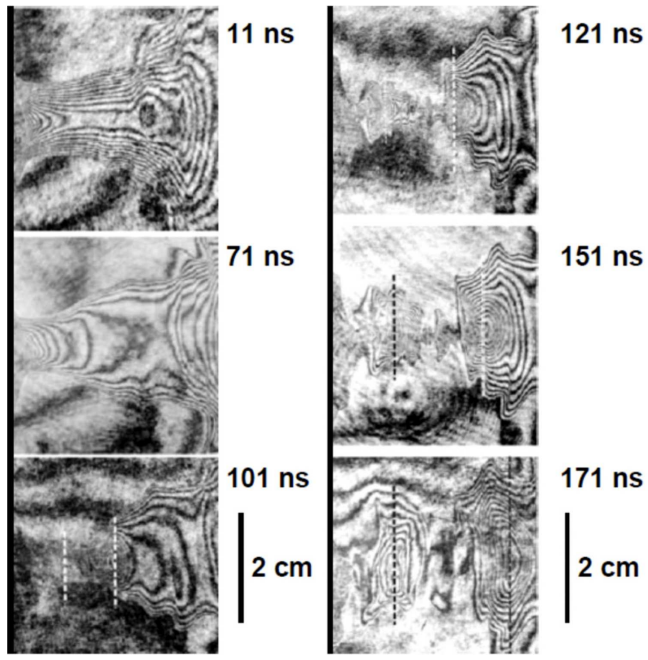

Fig. 3. Interferometric images of a discharge performed with a conical tip upon the anode-end, as recorded in different instants after the first current dip. The vertical black lines (left edges) correspond to the anode end, and the thin broken lines mark cross-sections of two distinct plasmoids.

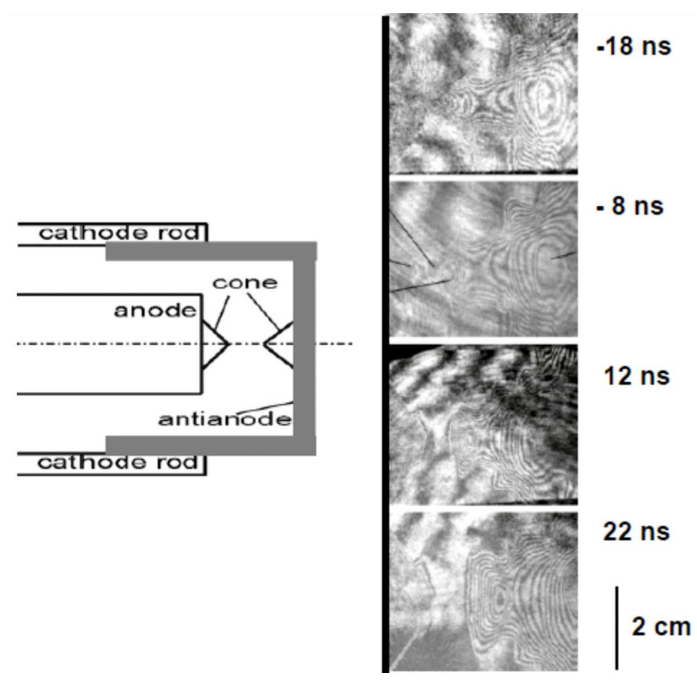

Fig. 4. Scheme of the PF-1000U electrodes with an anti-anode and some interferometric images which show the pinching before the current dip (at $-18 \mathrm{~ns}$ ). The vertical black line (left edge) shows the anode end and the thin black lines (at $-8 \mathrm{~ns}$ ) indicate several plasmoidal structures.

Other experiments in the PF-1000U facility concerned an influence of an anti-anode which was also equipped with a conical tip but oriented in the opposite direction and fixed to the copper plate connected with the cathode rods. The main aim of discharges performed with the anti-anode was to check a possibility of reducing the pinch axial dimensions during the radial compression of a current sheath [14]. A characteristic feature of shots with the anti-anode was that the pinch and the first hard X-ray pulse were observed about 20 ns before the current dip, as presented in Fig. 4. 
It was observed that the presence of the antianode strengthens a plasma jet emitted from the anode tip which is injected into the central pinch. In the described configuration during the discharges with a deuterium filling, a decay of the anode jet and the subsequent plasmoid evolution were also accompanied by the fusion-neutron emission, although the total yield was relatively lower. It means that the use of an anti-anode does nor help in the optimization of fusion reactions.

\section{Influence of gas conditions on plasma electron temperature}

The next research task was to study an influence of gas conditions on pinch parameters and, in particular, on the electron temperature $\left(T_{e}\right)$ inside the pinch column. The studies were performed with the basic anode configuration (see Fig. 1). Measurements of X-rays were carried out sideon with pinhole cameras equipped with sensitive X-ray films (for time-integrated observations) or filtered PIN-diodes (for time-resolved studies). Very soft X-rays were also recorded side-on by means of a XUV-camera [15, 16]. The investigated discharges in the PF-1000U facility were performed at a static initial gas-filling and at a pulsed injection of the working gas with (or without) another gas admixture. The PIN-diodes, which were located behind pinholes with different absorption filters and observed two regions (of about $3 \mathrm{~cm}$ in diameter at the pinch axis), delivered X-ray signals depending on local plasma parameters and the applied experimental conditions. From a ratio of the X-ray signals recorded behind Be-filters of different thickness, it was possible to estimate local values of the electron temperature $T_{e}$, as shown in Fig. 5 .

Detailed results of the studies were obtained at various initial gas conditions [15]. On the basis of the published data, it can be concluded that an admixture of a heavier gas facilitates the formation of plasma filaments and micro-regions of an increased X-ray emission (the so-called hot-spots).
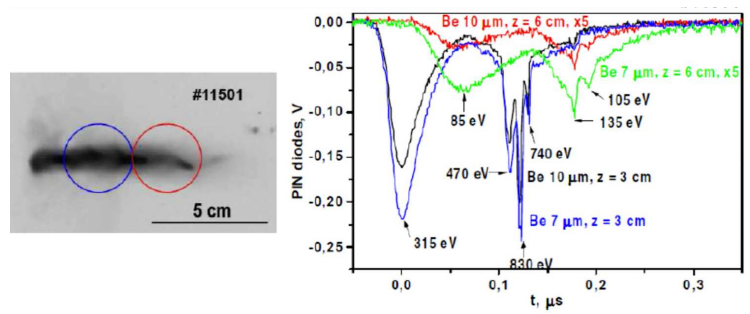

Fig. 5. X-ray pinhole image of a 300-kJ discharge performed at $\mathrm{p}_{0}=1.2 \mathrm{hPa}\left(\mathrm{D}_{2}+1 \% \mathrm{Ne}\right)$ and the additional axial injection of $\mathrm{D}_{2}$. Each region marked by a circle was observed by a couple of the PINdiodes with different filters. The diagram shows a comparison of X-ray signals from the PIN-diodes and $T_{e}$ values corresponding to the recorded peaks.
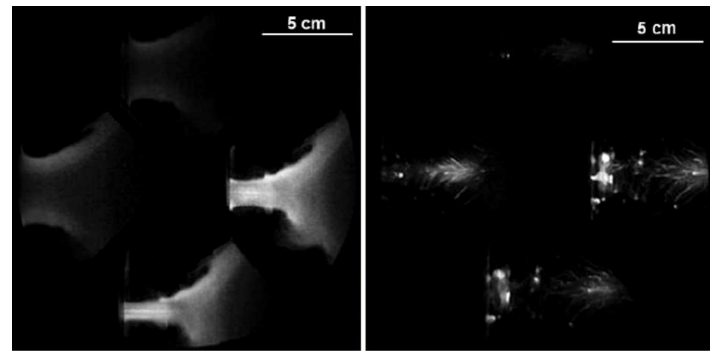

Fig. 6. Comparison of XUV images obtained from discharges performed at $p_{0}=1.2 \mathrm{hPa} \mathrm{D}_{2}$ (left) and at $p_{0}=1.1 \mathrm{hPa} \mathrm{D}_{2}+\mathrm{He}$ puffing (right). The frames visible in each image were recorded with a 2 -ns exposition at different instants: left frame at $52 \mathrm{~ns}$, bottom frame at $100 \mathrm{~ns}$ and right frame at $112 \mathrm{~ns}$.

Positions and dimensions of such plasma structures can be determined from X-ray pinhole images. In discharges at the pure $\mathrm{D}_{2}$-filling, the local $T_{e}$ values in the pinch column can amount to $150-200 \mathrm{eV}$, while in shots with a small admixture of a heavier noble gas (e.g. Ne) or with an additional injection of such a gas - more hot-spots appear and the local $T_{e}$ values can be higher and range from $2.2 \mathrm{keV}$ to $7.5 \mathrm{keV}$.

It should be noted that an influence of a heavier gas admixture (or its injection) on the appearance of many hot-spots has also been confirmed by XUV frames, as shown in Fig. 6.

\section{Generation of fast proton beams for boron experiments}

Another research task concerned the use of intense proton beams, which can be generated by PF-1000U discharges, for experiments with boron $\left({ }^{11} \mathrm{~B}\right)$ targets. Nuclear reactions $\left(\mathrm{p},{ }^{11} \mathrm{~B}\right)$ are of particular interest because they are fusion processes which do not produce fast neutrons. They emit only $\alpha$ particles which can easily be stopped and deposit their energy. Experiments on the realization of such reactions in the PF-1000U facility were initiated two years ago [12]. For this purpose, the facility was operated at the $\mathrm{H}_{2}$-filling and accelerated primary protons were applied for irradiations of a boron target $5 \times 5 \mathrm{~cm}^{2}$ which was placed at the angle of $45^{\circ}$ to the $z$-axis, at a distance of about $20 \mathrm{~cm}$ from the electrode outlets. In order to record $\alpha$ particles originating from $\left(\mathrm{p},{ }^{11} \mathrm{~B}\right)$ reactions, the use was made of nuclear track detectors of the CR-39 type, situated parallel to the target, at a distance of $5 \mathrm{~cm}$ from it but slightly shifted off the $z$-axis (to make the target irradiation possible). A scheme of the experimental set-up and a picture of the detector, which was exposed to proton streams and etched chemically after that irradiation, are presented in Fig. 7.

In the experiments performed in 2017, the proton streams emitted from the PF-1000U facility were measured [16] but $\alpha$ particles on the CR-39 detector could hardly be analyzed due to many tracks 


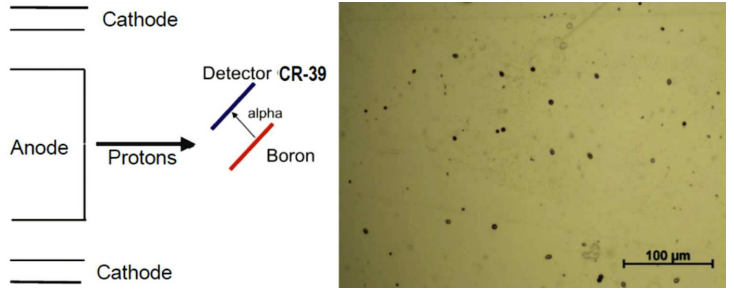

Fig. 7. Scheme of the experimental arrangement and a picture of the nuclear tracks which were obtained after one PF-1000U hydrogen shot and detector etching.

produced by other scattered ions. It was concluded that some shielding of the detector should be applied to considerably reduce the recording of the scattered ions. Therefore, a new detector arrangement has been designed and experiments on $\left(\mathrm{p}^{-11} \mathrm{~B}\right)$ reactions are to be continued soon.

\section{Generation of plasma jets for laboratory astrophysics}

Earlier experiments with different PF-type machines showed that high-current pulsed discharges, performed at appropriate gas conditions, can generate intense plasma streams and long plasma jets which can be used for simulation of astrophysical phenomena [17]. An example of such a plasma jet, which was produced within the PF-1000U facility, is shown in Fig. 8.

To investigate an influence of the initial gas conditions on parameters of plasma jets generated in the PF-1000U facility, after some preliminary studies [18], a new series of experiments was performed at various filling pressures $p_{0}\left(1.2 \mathrm{hPa} \mathrm{D}_{2}\right.$, $\left.1.06 \mathrm{hPa} \mathrm{D} \mathrm{D}_{2}+10 \% \mathrm{He}, \quad 0.53 \mathrm{hPa} \mathrm{D}+25 \% \mathrm{Ne}\right)$, without or with an additional gas puffing [19]. In discharges with the gas puffing, about $1 \mathrm{~cm}^{3}$ of pure $\mathrm{D}_{2}, \mathrm{He}$, Ne or a mixture $(50 \% \mathrm{He}+50 \% \mathrm{Ne})$, compressed to the pressure of $(0.13-0.20) \mathrm{MPa}$, was injected into the experimental chamber, about $1.5 \mathrm{~ms}$ before the main discharge initiation. To measure the propagation of the plasma front, three optical collimators placed side-on were applied at different distances from the electrode outlets and coupled with fast photomultipliers. In order to study plasma parameters, the use was made of a sideon quartz window and an optical collimator coupled with a Mechelle ${ }^{\circledR} 900$ spectrometer which observed a chosen region of a plasma jet. An example of the optical spectra, which were recorded at $z=27 \mathrm{~cm}$ for five $300-\mathrm{kJ}$ discharges with the gas puffing, is shown in Fig. 9.

Almost all the investigated discharges produced a dense plasma-focus column (of about $10 \mathrm{~cm}$ in length) and a long (up to about $100 \mathrm{~cm}$ ) plasma jet which existed for several $\mu \mathrm{s}$. The ambient plasma density at the pure deuterium filling was about $10^{16} \mathrm{~cm}^{-3}$ but in the cases of a $10 \% \mathrm{He}$ or $25 \% \mathrm{Ne}$ admixture it was higher by a factor of (1.8-2.5).

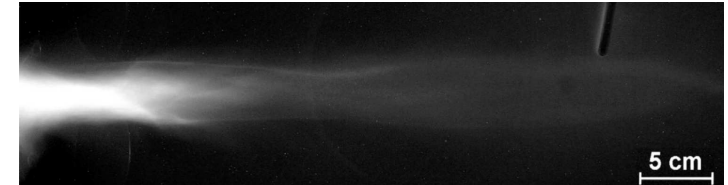

Fig. 8. Side-on picture of a long plasma jet producing a discharge within the PF-1000U machine at $p_{0}=1.2 \mathrm{hPa} \mathrm{D}, U_{0}=16 \mathrm{kV}$ and $I_{\max }=1.4 \mathrm{MA}$.

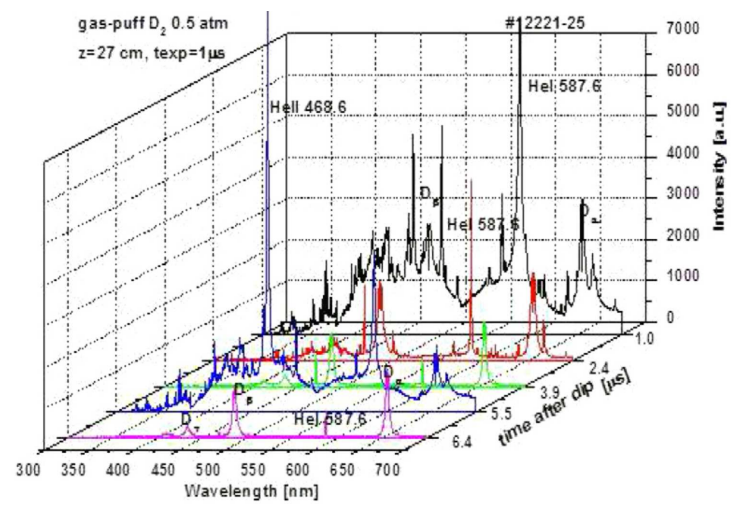

Fig. 9. Changes of the optical lines emission from PF-1000U shots as a function of a time delay after the discharge current peculiarity.

In all cases, the plasma jet density was more than 10 times higher than that of ambient plasma. At the He- or Ne-puffing, this density amounted to $(3.5-6) \times 10^{17} \mathrm{~cm}^{-3}$. The recent measurements were performed at a distance which was about twice as short as that during the preliminary studies but dimensionless parameters of the plasma jets (Mach numbers, the density contrast $n_{\text {jet }} / n_{\text {ambient }}$, Reynolds and Peclet numbers) were similar to those determined in the earlier studies [18]. Hence, it can be concluded that discharges in the PF-1000U facility at the determined gas conditions (particularly those with the gas puffing) might be applied for laboratory simulations of astrophysical plasma jets emitted from young stellar objects (YSO).

\section{Interaction of intense plasma streams with solid targets}

From the very beginning of research on PF-type discharges, it' was expected that intense plasma streams can be used for material studies. About 15 years ago, studies of plasma-material interactions in the PF-1000 (and later in the PF-1000U) facility were initiated using different solid samples [20-22]. Particular attention was focused on studies of materials which are of interest for fusion technology, i.e., graphite, carbon fibre composite (CFC), pure tungsten (W) and its alloys, Al-Mg-Li alloys, etc.

In 2018, a comparative analysis of W-surface damages, which were produced by powerful laser pulses or pulsed ion/plasma streams from a small PF-6 device [23], was performed. The erosion of 

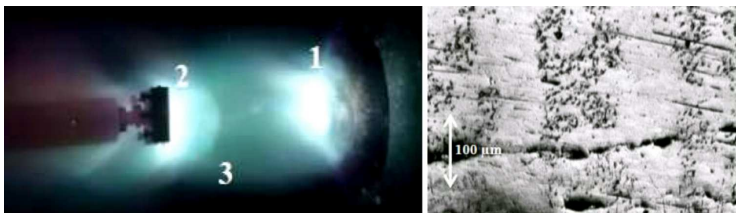

Fig. 10. Picture of a discharge taken during irradiation of the W-sample (left) which shows: 1 a primary deuterium plasma stream, 2 - secondary plasma from the target, 3 - a shock front created in the deuterium plasma stream, (right) and a SEM image of the cross-section perpendicular to the target surface which shows stratification and cracks induced by the irradiation.

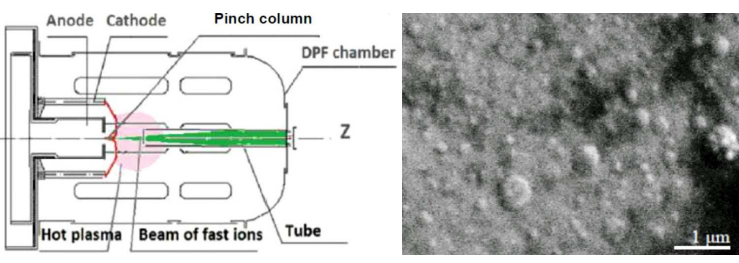

Fig. 11. Experimental arrangement for irradiations of the stainless-steel tube (left) and a microscope picture of the irradiated internal wall (right) which shows distinct blisters.

W-samples was also investigated in the PF-1000U facility operated with the anode equipped with a conical tip which had a central hole in order to reduce the evaporation of copper during PF discharges. The investigated $\mathrm{W}$-sample was placed in a holder at the electrode axis [24]. Its distance from the anode end was changed during different experiments from $7 \mathrm{~cm}$ (when the sample was immersed in about 10-cm-long pinch column) to $15 \mathrm{~cm}$. The sample was irradiated by a chosen number of shots. During the described experiments, a deuterium plasma density was $10^{18}-10^{19} \mathrm{~cm}^{-3}$ and a plasma flow velocity amounted to $3 \times 10^{7} \mathrm{~cm} / \mathrm{s}$. The power flux density of the plasma stream upon the $\mathrm{W}$-target was $10^{9}-10^{10} \mathrm{~W} / \mathrm{cm}^{2}$ and the duration of the plasma pulse was 50-100 ns. The power flux density of fast $(>100 \mathrm{keV})$ ions was estimated to be $10^{11}-10^{12} \mathrm{~W} / \mathrm{cm}^{2}$ and the duration of the fast ion pulse was about $10-50 \mathrm{~ns}$. In addition to the analysis of the irradiated sample surface, metallographic cross-sections of the investigated samples were also examined. The irradiated $\mathrm{W}$-target and the scattering electron microscope (SEM) image of the irradiated sample are shown in Fig. 10.

Further experiments at the PF-1000U facility were performed with a hexahedral tube made of a $25 \mathrm{Cr}_{12} \mathrm{Mn}_{20} \mathrm{~W}$ austenitic steel which had an average diameter of about $8 \mathrm{~cm}$ and was placed along the $z$-axis, at a distance of $12 \mathrm{~cm}$ from the anode end. In that case, only the internal wall of the tube was irradiated, since the most intense ion beams were emitted in a small spatial angle, as shown in Fig. 11.
It can be concluded that the reported experimental results proved that the PF-1000U is an useful tool for material engineering studies and different damages (melting, blistering, forming of bubbles, etc.) of the investigated materials can be observed, depending on a power flux density and other characteristics of the applied plasma streams.

\section{Characteristics \\ of the fast deuterons emission}

The emission of fast deuteron beams from PF-type discharges has been investigated [25-29] for many years but it has been of interest to study such beams in the PF-1000U facility at the present electrode configuration. In this facility, there were earlier observed complex structures of the pinch column and distinct plasma-current filaments [30] which might produce strong local magnetic fields and considerably deflect deuteron trajectories. Noticeable transformations of the ordered internal plasma structures during the emission of fast charged particles [31] were also observed. Therefore, during the recent experiments — in order to record fast ion beams (in particular deuterons) - the use was made of several pinhole cameras oriented at different angles to the discharge axis and placed on a semi-circular support at a distance of $32 \mathrm{~cm}$ from the anode outlet [32]. These cameras were equipped with PM-355 track detectors, shielded with Al-foils of an appropriate thickness which enabled to record deuterons above the chosen energy threshold. An example is presented in Fig. 12.

Simultaneously with measurements of the ion beams, characteristic waveforms and laserinterferometry images were recorded. From the analysis of the obtained experimental data it has been possible to conclude that the characteristics of the fast deuterons in the PF-1000U facility are very similar to those observed in earlier PF-experiments. The fast deuterons, which are not lost in the D-D fusion reactions, are emitted from the pinch column. Many deuteron beams are emitted along the discharge axis and they form quasi-circular images composed of numerous micro-beams. Energies of the fast deuteron beams usually range from about $100 \mathrm{keV}$ to several hundred $\mathrm{keV}$ but in many discharges some deuteron micro-beams of energy ranging around a few $\mathrm{MeV}$ can also be recorded.

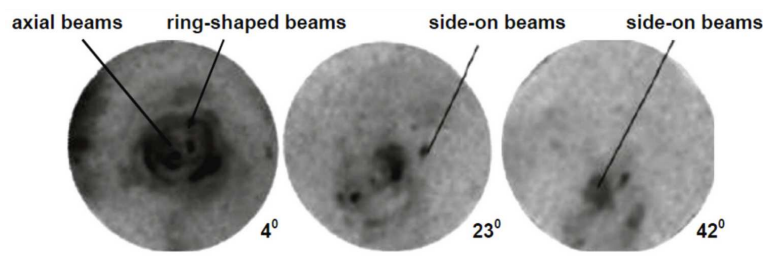

Fig. 12. Images of the fast deuteron beams emitted from a single 250-kJ PF-1000U discharge. 


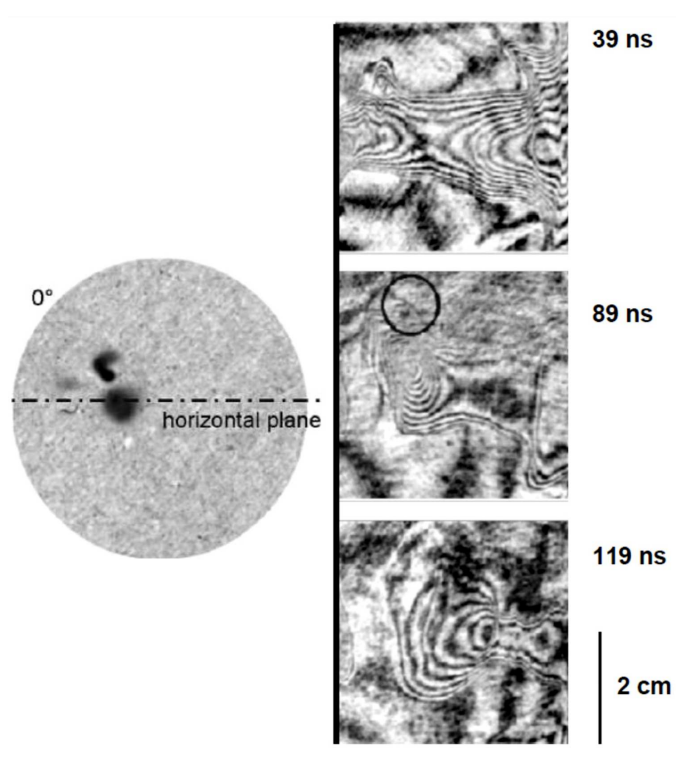

Fig. 13. Pinhole image of deuterons emitted from one PF-1000U shot along the $z$-axis (left) and interferometric images of the pinch column. The black vertical line (left edge) corresponds to the anode end and the black circle indicates the plasma region which might be responsible for the emission of the recorded asymmetrical ion emission.

Such micro-beams can be selected and recorded, e.g. with the use of appropriate absorption filters. A divergence of such micro-beams in the PF-1000U was not measured accurately but taking into account diameters of the recorded micro-beam spots and a distance from the pinch centre, it was estimated that this divergence was $<8^{\circ}$. The total amount of the recorded fast deuterons can be determined by counting individual tracks (visible under an optical microscope) upon the irradiated and etched detectors. Unfortunately, the obtained data are characterized by substantial jitter due to a strong anisotropy and irreproducibility of the ion emission from shot to shot. For the PF-1000U facility, basing on the ion images recorded for a series of discharges performed at about $260 \mathrm{~kJ}$ and the identical gas conditions, it was estimated that on average the total amount of the fast deuterons of energy exceeding $100 \mathrm{keV}$ (emitted in the whole front half-space) reached about $10^{16}-10^{17}$. It is well known that the energy distribution of the deuterons within the energy range from about $100 \mathrm{keV}$ to about $500 \mathrm{keV}$ can be approximated by the power law $\mathrm{d} N / \mathrm{d} E \sim E^{-\gamma}$, where $\gamma=3 \pm 1$, but it is not known whether this approximation can be used for very high-energy deuterons ( $>500 \mathrm{keV}$ ). Nevertheless, an energy input of these very high-energy deuterons can be neglected and the total energy of the considered deuteron beams can be estimated to be about $100-1000 \mathrm{~J}$. This is a small part of the energy amount delivered from the condenser bank but it is brought by the deuterons which were not lost in fusion reactions and were emitted

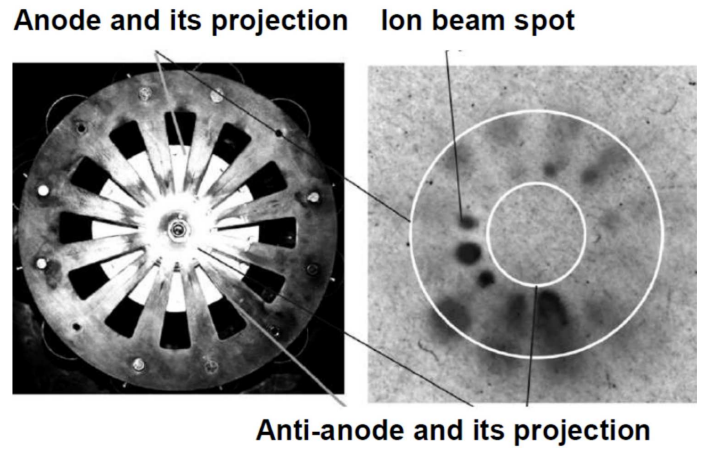

Fig. 14. Picture of the electrodes outlets with the installed anti-anode (seen from the $z$-axis) and an ion pinhole image obtained from three successive 260-kJ discharges. One can easily discern distinct spots, spots with pale centres and spots elongated in the radial direction.

from the discharge in the whole front half-space. It should be mentioned here that the deuteron beams recorded at larger angles can be explained as an emission from different regions of the pinch column, as shown in Fig. 13.

It should also be noted that the fast deuteron beams at the $z$-axis are weakly influenced by local magnetic fields of current filaments and other plasma internal structures but the deuterons emitted even at small angles to that axis can be strongly deflected by such local fields. The deuterons, which leave the pinch and penetrate the front currentsheath layer, can propagate along almost straight trajectories.

Recent studies of the fast deuteron beams in the PF-1000U facility have been performed with the axial pinhole camera for shots without and with the additional anti-anode [33]. The ion images recorded from shots without any anti-anode have shown the characteristic central deuteron spot and a ring-shaped region composed of many microbeams. The obtained ion images were analyzed with an optical microscope and energies of the recorded deuterons were estimated on the basis of the measured track dimensions. The estimated values appeared to be consistent with those from the earlier studies.

Some new results were provided by the recent PF-1000U experiment with the anti-anode which had no central hole but several radial slits of the triangular shape. In that case, the deuteron beams emitted along the $z$-axis were eliminated (stopped by the anti-anode central part) but those penetrating through slits could be recorded at a chosen distance, as presented in Fig. 14.

On the basis of the ion pinhole images (obtained end-on) and laser interferometric frames (recorded side-on), it could be deduced that the recorded deuteron spots corresponded temporally and spatially to some plasmoids and plasma lobules formed around the main pinch column. The recorded 
deuterons were of course deflected by local magnetic fields of internal current filaments and later on by external magnetic fields produced by the global current flows. The acceleration of the primary deuterons could be explained by local transformations of the magnetic energy into induced electrical fields but to confirm this hypothesis more sophisticated diagnostics and realistic numerical simulations are needed.

It should be noted that the main results of the studies described above have already been partially discussed in an invited paper [34] however there are still many questions to be analyzed. It particularly refers to the PF-1000U experiments which have been carried out recently.

\section{Evolution of a pinch during the emission of charged particles}

In the recent experimental campaigns at the PF-1000U facility, particular attention was focused on studies of the pinch evolution during the emission of fast ion-beams [35]. Numerous interferometric frames, as taken with the exposition time equal to about 2 ns and recorded side-on the pinch column at various instants of the studied discharges, were compared and analyzed. Voltage- and currentwaveforms, as well as signals of hard X-rays (originating from fast electrons) and pulses of energetic neutrons (produced by fusion reactions)

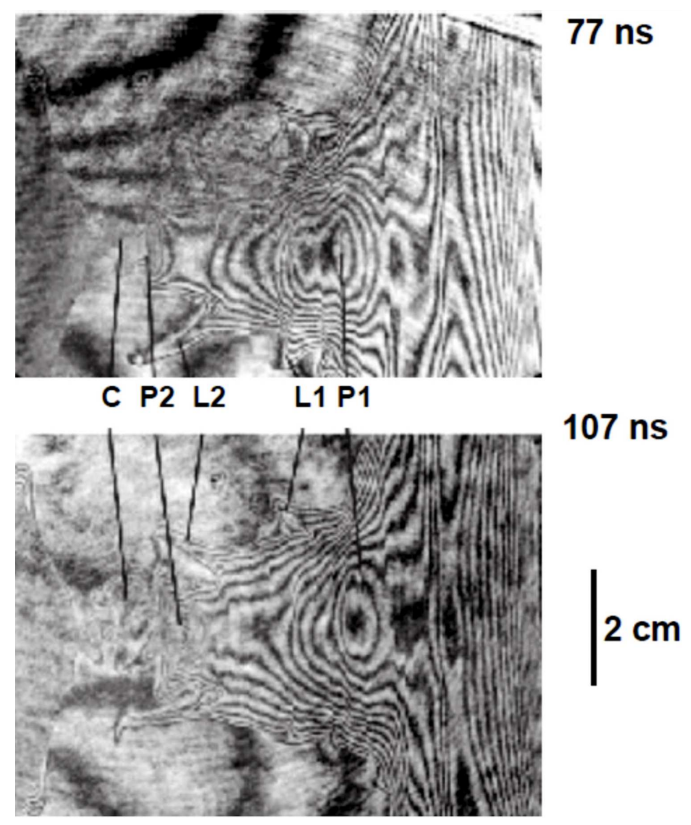

Fig. 15. Interferometric frames of the pinch column (with the anode end at the left edge) which were taken from a $350-\mathrm{kJ}$ shot at different instants after the first current dip: (upper) before HXR and neutron pulses, (bottom) during the HXR and neutron emission. The letters show: $\mathrm{C}-$ constriction, P1 and P2 - plasmoids, L1 and L2 plasma lobules.

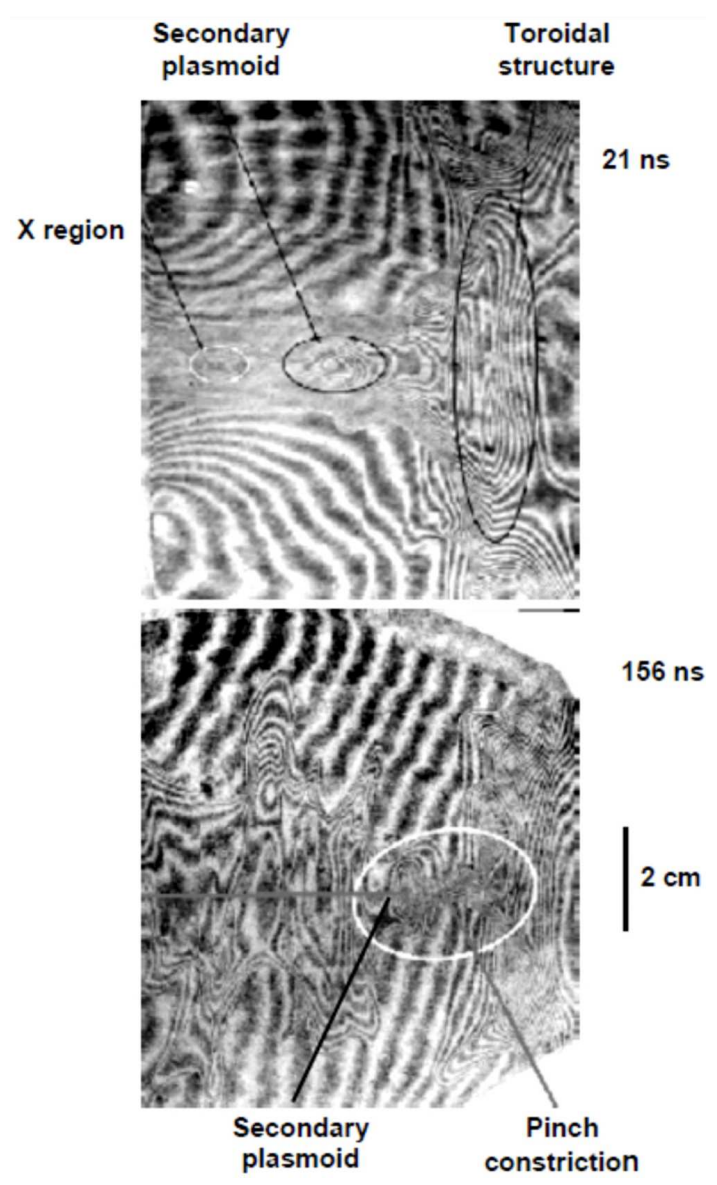

Fig. 16. Interferometric frames taken in different instants of two PF-1000U discharges which show characteristic internal plasma structures inside the pinch column.

were also recorded with the temporal resolution of about 4 ns. There were also recorded timeintegrated images of fast deuteron beams emitted mainly along the $z$-axis. The development of pinch constrictions, plasmoids and plasma lobules (upon the surface of the pinch column) was studied in detail, as shown in Fig. 15.

The experiments reported above have been continued during the subsequent session at the PF-1000U facility and numerous discharges (at the similar experimental conditions) were investigated in order to observe correlations of the charged particle emission with internal plasma structures and their dynamics [36]. Some examples of the typical interferometric images are presented in Fig. 16.

The described studies of many PF-1000U discharges documented the appearance of the toroidal and plasmoidal plasma-structures which could be formed by internal currents flowing inside the pinch column. The appearance of the corresponding poloidal and toroidal magnetic fields, which could undergo different transformations, was confirmed. It could be deduced that the observed reconnections of the internal currents (filaments) induce transformations of magnetic energy into kinetic energy of 
the accelerated particles. It could be concluded that the fast deuterons can be accelerated in various developing and decaying pinch constrictions, plasmoids and plasma lobules, while the fusion reactions (producing fast neutrons) can occur in dense plasma existing nearby, e.g. in neighbouring plasmoids.

\section{Summary and conclusions}

The most important achievements of recent plasma studies in the PF-1000U facility can be summarized as follows:

1. New experimental data about the influence of the anode-end and anti-anode on discharge dynamics and emission characteristics were collected and the application of a conical anode-tip improved the formation of a plasma column and the total neutron yield.

2. The dependence of plasma electron temperatures on gas conditions was investigated and high local $T_{e}$ values (ranging between 5 and $8 \mathrm{keV}$ ) were measured in the so-called hotspots.

3. The possibility of generation of fast proton beams by hydrogen discharges was proved and the production of $\alpha$ particles from $\left(\mathrm{p}^{\left.-{ }^{11} \mathrm{~B}\right)}\right.$ reactions was tested.

4. The generation of long plasma jets for modeling of astrophysical phenomena was investigated as a function of gas conditions.

5. The PF-1000U facility was used for studies of interaction of intense plasma streams with different solid materials, e.g. tungsten and its alloys, stainless steel, etc.

6. Characteristics of fast deuteron emission were investigated and their relation with the operational conditions was determined.

7. The evolution of the pinch column during the emission of charged particles (i.e., fast electrons and ions) was studied in detail.

As the first conclusion, it might be stated that experimental and theoretical studies of dense plasma discharges within the PF-1000U facility should be continued due to their importance for dense plasma physics and numerous applications. Such studies might be supervised by experts from the ICDMP Scientific Committee.

The second conclusion is that research on mechanisms and sources of the fusion neutrons emission in correlation with other plasma characteristics is a very important issue.

The third conclusion is a proposal that the laser interferometry should be supplemented not only by the time-integrated ion detection but also by timeresolved measurements of the fast deuteron beams. Such measurements could be performed, e.g. with miniature detectors placed in different places on the image plane of the applied pinhole camera or with a modern micro-channel plate supplied by appropriate electronic units.

\section{Acknowledgments}

The reported studies of PF-1000U discharge characteristics have been supported in part by the Research Program under Grants Nos. MSMT CZ.02.1.01/0.0/0.0/16 019/0000778, MSMT LTT17015, LTAUSĀ17084, GACR 16-07036S, IAEA CRP RC-19253, SGS 16/223/OHK3/3T/13 and by the Polish Ministry of Science and Higher Education in the framework of the financial resources allocated in 2016-2018 for the realization of international co-financed projects. The study of plasma jets has additionally been supported by a grant of the Russian Foundation for Basic Research \#18-29-21006 as well as by Grants Nos. IAEA RC-16115, RC-16954, RC-16956, RC-17088 and RC-19253. The material studies have been supported in the frame of the IAEA CRP F13016 under Contract No. 19253 and were carried out according to the R.F. State Assignment No. 007-00129-18-00. The authors also wish to express thanks to all their colleagues participating in the described studies. Their names are given in the references cited below.

\section{References}

[1] M. Scholz, L. Karpiński, M. Paduch, K. Tomaszewski, R. Miklaszewski, T. Pisarczyk, M.J. Sadowski, A. Szydłowski, A.V. Dubrovski, I.E. Volobujev, Czech. J. Phys. 50 Suppl. S3, 179 (2000).

[2] M.J. Sadowski, M. Scholz, Nukleonika 47, 31 (2002).

[3] M. Borowiecki, B. Bienkowska, S. Jednorog, L. Karpinski, M. Paduch, M. Scholz, M.J. Sadowski, Czech. J. Phys. 56, B184 (2006).

[4] M. Scholz, B. Bienkowska, M. Borowiecki et al., Nukleonika 51, 79 (2006).

[5] V.A. Gribkov, A. Banaszak, B. Bienkowska et al., J. Phys. D: Appl. Phys. 40, 3592 (2007).

[6] P. Kubes, J. Kravarik, D. Klir et al., IEEE Trans. Plasma Sci. 37, 83 (2009).

[7] P. Kubes, M. Paduch, T. Pisarczyk et al., IEEE Trans. Plasma Sci. 38, 672 (2010).

[8] P. Kubes, D. Klir, M. Paduch et al., IEEE Trans. Plasma Sci. 40, 1075 (2012).

[9] M. Kubkowska, E. Składnik-Sadowska, R. Kwiatkowski et al., Phys. Scripta T161, 014038 (2014).

[10] P. Kubes, M. Paduch, J. Cithardt et al., Phys. Plasmas 21, 082706 (2014).

[11] P. Kubes, M. Paduch, B. Cikhardtova et al., Phys. Plasmas 23, 112708 (2016).

[12] M. Paduch, R. Miklaszewski, in: Proc. ICDMP-2016 Workshop, IPPLM, Warsaw 2016. 
[13] P. Kubes, M. Paduch, J. Cikhardt et al., Phys. Plasmas 24, 092707 (2017).

[14] P. Kubes, M. Paduch, M.J. Sadowski et al., Phys. Plasmas 25, 062712 (2018).

[15] D.R. Zaloga, M.J. Sadowski, E. SkladnikSadowska, M. Paduch, E. Zielinska, K. Tomaszewski, J. Phys.: Conf. Ser. 959, 012003 (2018).

[16] A. Szydlowski, R. Kwiatkowski, A. Malinowska, M. Paduch, R. Miklaszewski, E. Zielinska, in: Proc. Intern. Conf. PLASMA-2017, IPPLM, Warsaw 2017.

[17] V.I. Krauz, V.V. Myalton, V.P. Vinogradov et al., J. Phys.: Conf. Ser. 907, 012026 (2017).

[18] E. Skladnik-Sadowska, S.A. Dan'ko, R. Kwiatkowski, M.J. Sadowski, D.R. Zaloga, M. Paduch, E. Zielinska, A.M. Kharrasov, V.I. Krauz, Phys. Plasmas 23, 122902 (2016).

[19] E. Skladnik-Sadowska, S.A. Dan'ko, A.M. Kharrasov, V.I. Krauz, R. Kwiatkowski, M. Paduch, M.J. Sadowski, D.R. Zaloga, E. Zielinska, Phys. Plasmas 25, 082715 (2018).

[20] V.A. Gribkov, A.V. Dubrovsky, K. Malinowski et al., Czech J. Phys. 56, 1401 (2006).

[21] M.J. Sadowski, V.A. Gribkov, P. Kubes, K. Malinowski, E. Składnik-Sadowska, M. Scholz, A. Tsarenko, J. Żebrowski, Physica Scipta T123, 66 (2006).

[22] V.A. Gribkov, Ye.S. Grebenschikova, Ye.V. Demina et al., American Institute of Physics CP 993, 361 (2008).

[23] V.A. Gribkov, M. Paduch, E. Zielinska, A.S. Demin, E.V. Demina, E.E. Kazilin, S.V. Latyshev, S.A. Maslyaev, E.V. Morozov, V.N. Pimenov, Radiat. Phys. and Chemistry 150, 20 (2018).
[24] E. Kowalska-Strzeciwilk, V.A. Gribkov, TECDOC-IPPLM Report 2018, IPPLM, Warsaw 2018.

[25] L. Bertalot, H. Herold, U. Jager, A. Mozer, T. Oppenlander, M. Sadowski, H. Schmidt, Phys. Letters 79A, 389 (1980).

[26] A. Mozer, M. Sadowski, H. Herold, H. Schmidt, J. Appl. Phys. 53, 2959 (1982).

[27] M. Sadowski, J. Żebrowski, E. Rydygier, H. Herold, U. Jager, H. Schmidt, Phys. Letters 113A, 25 (1985).

[28] A. Szydlowski, M. Sadowski, M. Scholz, J. Techn. Phys. 39 Spec. Suppl., 73 (1998).

[29] A. Bernard, H. Bruzzone, P. Choi et al., J. Moscow Phys. Soc. 8, 93 (1998).

[30] P. Kubes, M. Paduch, J. Cikhardt et al., Phys. Plasmas 24, 032706 (2017).

[31] P. Kubes, M. Paduch, J. Cikhardt et al., Phys. Plasmas 24, 072706 (2017).

[32] P. Kubes, M. Paduch. M.J. Sadowski et al., Phys. Plasmas 25, 012712 (2018).

[33] P. Kubes, M. Paduch, M.J. Sadowski et al., Phys. Plasmas 26, 032702 (2019).

[34] M.J. Sadowski, Ser. Plasma Phys. 6, 121 (2018).

[35] P. Kubes, M. Paduch, M.J. Sadowski et al., J. Fusion Energy 38, 490 (2019).

[36] P. Kubes, M. Paduch, M.J. Sadowski et al., IEEE Trans. Plasma Sci. 47, 339 (2019). 